\title{
Caractéristiques des ressources fourragères herbacées des pâturages naturels du terroir de Vipalogo (Burkina Faso)
}

\author{
Georges YAMEOGO ${ }^{1}$, André KIEMA ${ }^{2}$, Barthélémy YELEMOU ${ }^{3 *}$ et \\ Lucien OUEDRAOGO ${ }^{4}$ \\ ${ }^{1}$ Institut de l'Environnement et de Recherches Agricoles (INERA), Cellule de Télédétection et d'Information \\ Géographique (CTIG) 01 BP 476 Ouagadougou, Burkina Faso. \\ ${ }^{2}$ Institut de l'Environnement et de Recherches Agricoles, DRREA de Dori. \\ ${ }^{3}$ Institut de l'Environnement et de Recherches Agricoles, DRREA de Saria, BP 10 Koudougou. \\ ${ }^{4}$ Institut de l'Environnement et de Recherches Agricoles, CREAF de Kamboinsé : 01 BP 476 Ouagadougou 01, \\ Burkina Faso. \\ *Corresponding author; E-mail: yelbart@hotmail.com; georges.yameogo@yahoo.fr
}

\section{RÉSUMÉ}

Pour contribuer à l'élaboration d'un système d'exploitation durable des ressources pastorales dans le terroir de Vipalogo, un inventaire floristique de la végétation herbacée associé à une évaluation de la phytomasse épigée a été réalisé au niveau de 3 parcours naturels représentés par les buttes cuirassées (unité 1), les glacis versants (unité 2) et les bas - glacis (unité 4). Les relevés floristiques effectués par la méthode des points quadrats linéaires ont permis d'identifier 104 espèces herbacées sur l'ensemble des 3 unités de parcours, avec 25 espèces communes. Le nombre de taxons herbacés recensés a été de 36 dans les relevés des buttes cuirassées, de 78 dans les relevés des glacis versants et de 80 dans ceux des bas-glacis. La contribution spécifique (CS en \%) des espèces a montré dans toutes les unités de parcours étudiées un fort pourcentage pour les Poaceae, les faibles pourcentages étant enregistrés au niveau des Cyperaceae. La capacité de charge est moins élevée dans les buttes cuirassées (2205 kg de MS/ha) à plus élevée dans les bas-glacis (3334,7 kg de M.S/ha). De cette étude, il se dégage la nécessité d'avoir un modèle de gestion des parcours qui intègre la végétation naturelle et la production de fourrage dans les exploitations.

() 2013 International Formulae Group. All rights reserved.

Mots clés : Unité géomorphologique, Végétation herbacée, Valeur pastorale, Phytomasse, Capacité de charge, Burkina Faso.

\section{INTRODUCTION}

Au Burkina Faso, la question de la gestion durable des ressources naturelles constitue aujourd'hui l'une des préoccupations majeures aussi bien des chercheurs, des développeurs que des producteurs. En effet, les ressources naturelles subissent actuellement une dégradation très prononcée dans de nombreux terroirs villageois du pays (Millogo-Rasolodimby 2000 ; Yélémou et al., 2007 ; Sawadogo et al., 2012). La pression démographique, l'amélioration des systèmes de production et l'introduction des cultures de rente ont favorisé l'extension des champs de cultures au détriment des aires de pâture constituées par les jachères (Yaméogo, 1997) et les formations naturelles. Les conséquences en 
sont le surpâturage, l'extension des zones dénudées et la diminution des espèces fourragères les plus appétées.

Le terroir de Vipalogo, notre site de recherche n'échappe pas à cette situation de détérioration des ressources naturelles. L'élevage y est pratiqué de façon extensive et les ressources fourragères au lieu d'être produites et entretenues par l'Homme, proviennent essentiellement de la végétation naturelle soumise à l'action de nombreux facteurs anthropiques et écologiques (BotoniLiehoun et al., 2006).

Face à cette situation et par soucis de contribuer à une meilleure gestion des aires de production agro-sylvo-pastorales, il a été entrepris dans le terroir, une étude sur la végétation naturelle herbacée dont l'objectif global est d'aboutir à l'élaboration d'un système d'exploitation durable des ressources naturelles disponibles. Elle a consisté à caractériser la végétation herbacée des parcours sylvo-pastoraux sur le plan qualitatif (composition floristique, valeur pastorale) et quantitatif (productivité) afin de préciser les potentialités pastorales du terroir en terme de charge réelle. Le présent article qui fait l'économie des résultats obtenus s'articule sur les points suivants : la présentation de la zone d'étude, le matériel et méthode, les résultats et la discussion.

\section{MATERIEL ET METHODES \\ Présentation de la zone d'étude}

L'étude a été réalisée à Vipalogo, un village situé à $40 \mathrm{~km}$ au Sud-Ouest de Ouagadougou (capitale du Burkina Faso et chef-lieu de la province du Kadiogo). Il est compris entre la latitude $12^{\circ} 04^{\prime} 20^{\prime}$ ' et $12^{\circ} 09^{\prime} 30^{\prime \prime}$ nord et $1^{\circ} 42^{\prime} 50^{\prime \prime}$ et $1^{\circ} 49^{\prime} 00^{\prime \prime}$ ouest (Figure 1).

Vipalogo appartient au domaine phytogéographique Nord-Soudanien caractérisé par des savanes arborées et arbustives à Vitellaria paradoxa C.F. Gaertn., Lannea microcarpa Engl. \& K. Krause, Parkia biglobosa (Jacq.) R.Br. ex G. Don., Faidherbia albida (Del.) Chev., Tamarindus indica L., Combretum micranthum G. Don,
Combretum glutinosum Perr. ex DC., Guiera senegalensis J.F. Gmel., Acacia seyal Del (Fontes et Guinko, 1995). Le paysage est marqué par la présence de savanes parcs et jachères à karité (Vitellaria paradoxa) et à néré (Parkia biglobosa).

Le climat est caractérisé par une saison sèche de sept mois (de novembre à mai) et une saison pluvieuse de cinq mois (de juin à octobre). La pluviométrie annuelle très variable, oscille entre 600 et $1000 \mathrm{~mm}$, avec une moyenne de $761 \mathrm{~mm}$ sur les 30 dernières années. Les températures moyennes mensuelles connaissent également une variation allant de 24 à $33{ }^{\circ} \mathrm{C}$.

Le terroir de Vipalogo fait partie du grand ensemble généralement appelé «Plateau Central» qui se caractérise par une densité de population de 80,9 habitants au $\mathrm{km}^{2}$, contre une moyenne nationale de 51,8 habitants au $\mathrm{km}^{2}$ (INSD, 2009). Cette région est aussi marquée par une intense occupation qui entraîne la dégradation des terres et des conflits assez fréquents entre agriculteurs et entre agriculteurs et éleveurs.

Dans le terroir, six (06) unités géomorphologiques d'une superficie totale de 5278 ha sont identifiées et cartographiées (Figure 2). Ce sont: les glacis versants $(49,3 \%)$, les bas glacis $(27,2 \%)$, les bas glacis cuirassés $(11,3 \%)$ les buttes cuirassées $(8,2 \%)$ les bas-fonds $(3,4 \%)$ et les glacis érodés (0,5\%) (Yaméogo, 2009).

\section{Etude de la végétation \\ Echantillonnage}

A partir de la carte des unités géomorphologiques du terroir de Vipalogo, les parcelles étudiées correspondent aux buttes cuirassées (unité 1), aux glacis versants (unité 2) et aux bas glacis (unité 4) soit $84,8 \%$ du terroir villageois. Deux relevés couvrant chacun une superficie de $2500 \mathrm{~m}^{2}$ (Zoungrana, 1991) ont été réalisés sur chacune des trois premières unités. Les autres unités les glacis cuirassées (unité 3), les glacis érodés (unité 5), les bas-fonds (unité 6) sont des zones majoritairement occupées par les champs ou 
impropres à toutes formes d'activités agricoles ou pastorales.

\section{L'inventaire floristique}

Il a été réalisé par la méthode des points quadrats alignés de Daget et Poissonet, (1971) en fin de période active de végétation au mois de septembre. En rappel, elle consiste à recenser la présence des espèces à la verticale des points disposés régulièrement à chaque $20 \mathrm{~cm}$ le long d'un double décamètre (20 m) tendu au dessus d'un tapis herbacée. Une tige métallique effilée matérialisant la ligne de visée est placée à chaque point, et les espèces en contact avec la tige sont recensées. Par convention, chaque espèce n'est recensée qu'une seule fois par ligne de visée. Elle permet de dresser la liste floristique des espèces rencontrées dans les unités végétales et de déterminer la fréquence de chacune d'elle. Sur le terrain, une fiche d'enregistrement des données sur laquelle en début de ligne étaient notés les noms des espèces annoncées (en contact avec la tige métallique) a été utilisée. Chaque colonne est consacrée à un point du double décamètre et les espèces annoncées étaient cochées par une croix dans la case correspondante.

La méthode permet ainsi de calculer: (i) la fréquence spécifique (FS), valeur absolue, correspondant à la proportion des espèces au niveau du sol; (ii) la contribution spécifique $\left(\mathrm{CS}_{\mathrm{i}}\right)$ de chaque espèce définie comme le rapport de la fréquence spécifique (FSi) de cette espèce à la somme des $\mathrm{FS}_{\mathrm{i}}$ de toutes les espèces recensées sur 100 points échantillonnés.

$C S_{i}=\frac{F S_{i}}{\sum_{i=1}^{n} F S_{i}} * 100 \quad$ Avec $\mathrm{n}=$ le nombre des espèces.

$\mathrm{CS}_{\mathrm{i}}$ et $\mathrm{FS}_{\mathrm{i}}$ sont les contributions et fréquences de l'espèce, $\mathrm{i}$ et $\mathrm{n}$ le nombre d'espèces. $\mathrm{CS}_{\mathrm{i}}$ est la fréquence relative de l'espèce i dans l'ensemble des fréquences spécifiques observées. La détermination du nombre d'observations est effectuée par le calcul de l'intervalle de confiance à partir de l'effectif cumulé ligne par ligne des contacts de l'espèce dominante sur l'effectif cumulé des contacts enregistrés par l'ensemble des espèces (Daget et Poissonet, 1971).
$I C= \pm 2 \sqrt{\frac{n(N-n)}{N^{3}}}$

«N» est l'effectif cumulé des contacts de l'ensemble des espèces, «n $»$ l'effectif cumulé des contacts de l'espèce dominante.

En plus, en vue de compléter les listes floristiques, des relevés de parcours (recensement systématique des espèces) ont été effectués sur chaque unité.

\section{La valeur pastorale}

$\mathrm{La}$ valeur pastorale brute $(\mathrm{VPb})$ a été calculée à partir de la composition floristique, la contribution spécifique (CS) et l'indice de qualité spécifique (IS) des espèces. Les valeurs de l'Indice de qualité spécifique des espèces ont été établies à partir d'une échelle de cotation de 0 à 5 à l'issue de la synthèse de nombreux travaux (Grouzis, 1979; Zoungrana, 1991 ; Kiema, 1994; Kaboré Zoungrana, 1995).

Formule de la valeur pastorale :

$\mathrm{VPb}\left(\%\right.$.) $=0,2 \sum$ (Csi x ISi)

\section{La phytomasse épigée et capacité de charge}

L'évaluation de la phytomasse épigée des herbacées a été faite par la méthode de la récolte intégrale sur des surfaces unitaires de 1 $\mathrm{m}^{2}$ au mois de septembre en fin de période maximum d'épiaison de la végétation. $\mathrm{Au}$ total, dix (10) répétitions de $1 \mathrm{~m}^{2}$ chacune ont été réalisées sur chaque unité. La matière sèche (MS) a été déterminée sur des échantillons de $500 \mathrm{~g}$ mis à l'étuve à $85{ }^{\circ} \mathrm{C}$ jusqu'à obtention de poids constituant (Levang et Grouzis, 1980 ; Fournier, 1982). Les capacités de charge ont ainsi été calculées à partir des données de la phytomasse épigée de chaque unité de parcours (Boudet 1984).

\section{Bilan fourrager}

Un inventaire systématique de toutes les exploitations du terroir a eu pour objet de nombrer les effectifs du cheptel bovin, ovin et caprin du terroir. Ce travail a permis sur la base des normes généralement admises en milieu tropical selon Boudet (1991) d'estimer la charge réelle à savoir :

- l'UBT est un bovin adulte de $250 \mathrm{~kg}$ de poids vif, dont les besoins d'entretien sont estimés à 2,5 kg par jour pour $100 \mathrm{~kg}$ de poids 
vif, soit $6,25 \mathrm{~kg}$ de matières sèches ingérable par jour ;

- un (01) bovin sahélien équivaut à 0,85 UBT et ;

- un (01) ovin ou un (01) caprin représente 0,12 UBT.

Le bilan fourrager a été calculé sur la base de cet inventaire et de l'estimation de la phytomasse. La formule du bilan fourrager est :

\section{$\mathrm{BF}=\mathrm{CCh}-\mathrm{CR}$}

$\mathrm{BF}=$ bilan fourrager $; \mathrm{CCh}=$ Capacité de charge des herbacées; $\mathrm{CR}=$ charge réelle sur les terroirs

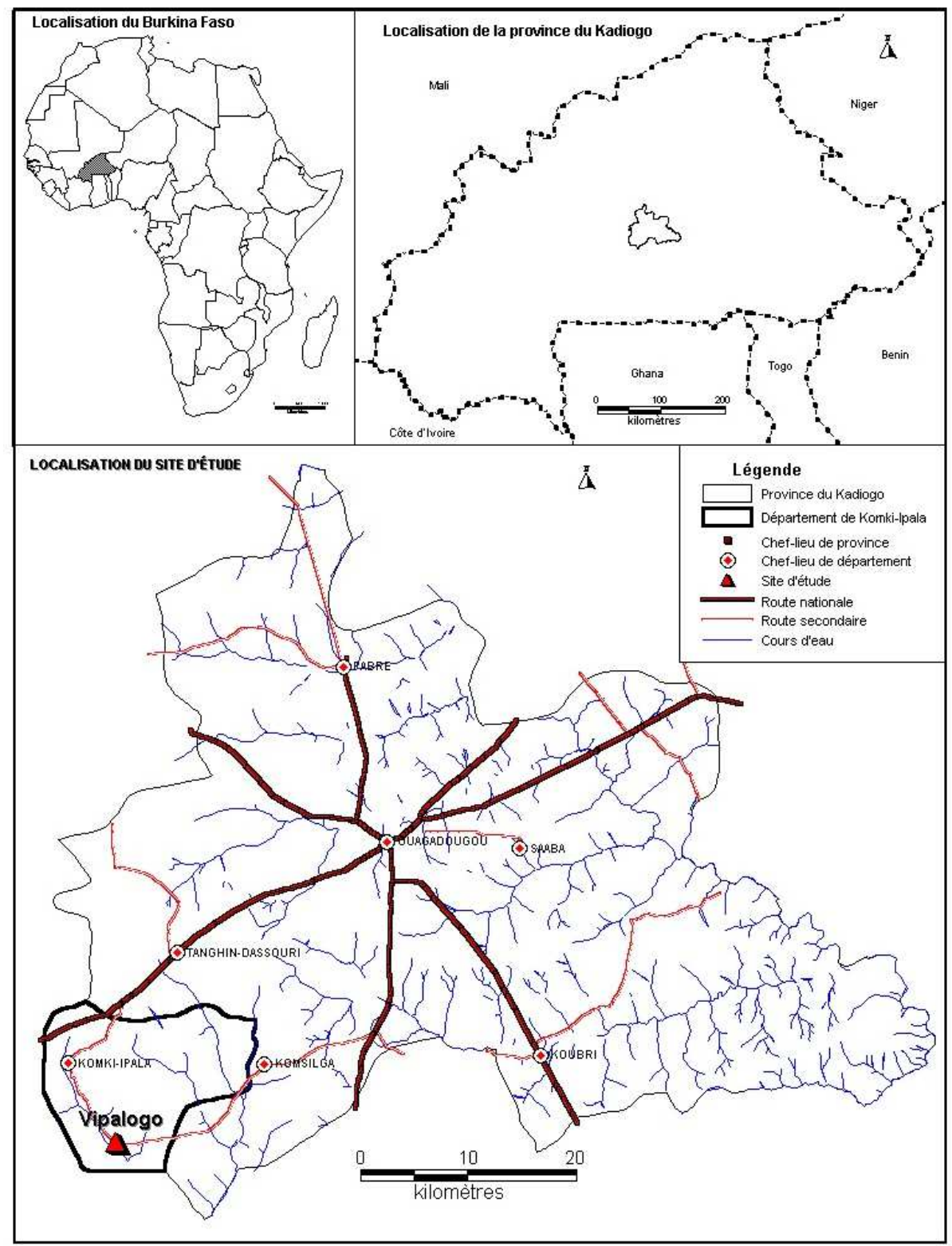

Figure 1 : Localisation du site de Vipalogo. Source : Yaméogo 2009. 


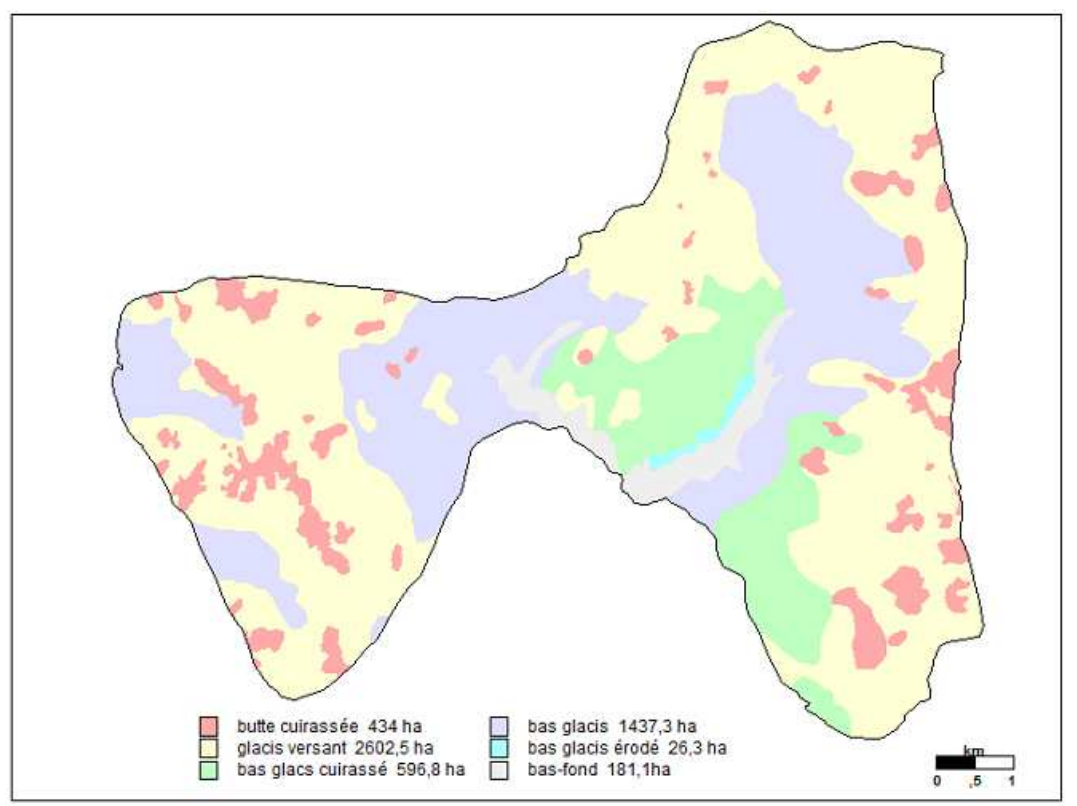

Figure 2 : Les unités géomorphologiques du terroir de Vipalogo. Source : Yaméogo 2009.

\section{RESULTATS}

\section{Composition floristique de la végétation herbacée \\ Diversité spécifique}

L'analyse de la composition floristique a permis d'identifier 104 espèces herbacées différentes sur l'ensemble des relevés effectués répartis dans 28 familles (Tableau 1). Au niveau des buttes cuirassées, 26 genres et 36 espèces ont été dénombrés. Le nombre le plus élevé de genres et d'espèces a été enregistré sur les parcours des glacis versants (56 genres et 78 espèces) et des bas glacis (63 genres et 80 espèces). Un total de 25 espèces communes est recensé sur les 3 unités de parcours.

Pour l'analyse, les espèces ont été regroupées en quatre catégories fourragères que sont: les Poaceae, les Légumineuses, les Cyperaceae et autres herbacées. Le Tableau 2 indique le nombre d'espèces de ces catégories fourragères par type de parcours.

\section{Contribution spécifique}

L'évaluation de la composition floristique et le recouvrement ont été appréciés par le calcul des fréquences spécifiques et des contributions spécifiques des espèces. Le Tableau 3 fait la synthèse des résultats de la contribution spécifique des espèces regroupées par catégories fourragères. Dans toutes les unités inventoriées, les poaceae avec 46,7 à $59,6 \%$ présentent les plus forts pourcentages de contribution spécifique sur toutes les unités.

Les espèces classées dans la catégorie «Autres herbacées », ont une contribution spécifique plus élevée que les légumineuses herbacées recensées.

\section{Valeur pastorale (VP)}

La valeur pastorale brute calculée est plus élevée au niveau des buttes cuirassées $(32,4 \%)$ qu'au niveau des glacis versants $(30,4 \%)$ et des bas glacis $(29,1 \%)$. Les valeurs obtenues pour les différentes catégories d'espèces sont présentées par le Tableau 4. Cette valeur est plus élevée pour les Poaceae $(20,5 \%$ à $23,8 \%)$, les légumineuses $(3,2 \%$ à $5,7 \%$ ) et est pratiquement nulle pour les Cyperaceae $(0 \%$ à $0,1 \%)$. 
Tableau 1: Récapitulatif des espèces présentes dans les unités.

\begin{tabular}{|c|c|c|c|c|}
\hline Espèces & Familles & $\begin{array}{l}\text { Buttes } \\
\text { Cuirassées }\end{array}$ & $\begin{array}{l}\text { Glacis } \\
\text { versants }\end{array}$ & Bas glacis \\
\hline Achyrantes aspera $L$. & Amaranthaceae & + & - & + \\
\hline Alysicarpus glumaceus (Vahl) DC. & Fabaceae & + & - & + \\
\hline Alysicarpus ovalifolius (Schum. et Thonn.) J. Leon. & Fabaceae & + & + & + \\
\hline Ampelocissus grantii (Bak.) Planch. & Ampelidaceae & - & - & + \\
\hline Andropogon ascinodis C.B.Cl. & Poaceae & + & - & + \\
\hline Andropogon fastigiatus Sw. Prod. & Poaceae & + & + & + \\
\hline Andropogon gayanus Kunth. & Poaceae & + & + & + \\
\hline Andropogon pseudapricus Stapf. & Poaceae & + & + & + \\
\hline Andropogon schirensis Hochst. ex A. Rich. & Poaceae & + & + & - \\
\hline Aristida kerstingii Pilger & Poaceae & - & - & + \\
\hline Aspilia bussei O. Hoffm. et Muschl. & Asteraceae & + & - & + \\
\hline Biophytum petersianum Klotz & Oxalidaceae & - & + & + \\
\hline Borreria radiata DC. & Rubiaceae & + & + & + \\
\hline Borreria scabra DC. & Rubiaceae & - & + & - \\
\hline Borreria stachydea (DC) Hutch. et Dalz. & Rubiaceae & + & + & + \\
\hline Brachiaria distichophylla (Trin.) Stapf & Poaceae & + & + & + \\
\hline Bulbostylis abortiva (Steud) C. B. Cl. & Cyperaceae & - & - & + \\
\hline Cassia mimosoides $\mathrm{L}$. & Caesalpiniaceae & + & + & + \\
\hline Cassia nigricans Vahl. & Caesalpiniaceae & + & + & + \\
\hline Cassia tora $\mathrm{L}$. & Caesalpiniaceae & - & + & - \\
\hline Chloris pilosa Schum. & Poaceae & - & - & + \\
\hline Cochlospermum planchonii Hook. F. & Cochlospermaceae & + & - & + \\
\hline Cochlospermum tinctorium A. Rich. & Cochlospermaceae & + & + & + \\
\hline Commelina forskalei Vahl. & $\begin{array}{l}\text { Commelinaceae } \\
2083\end{array}$ & - & + & + \\
\hline
\end{tabular}


G. YAMÉOGO et al. / Int. J. Biol. Chem. Sci. 7(5): 2078-2091, 2013

\begin{tabular}{|c|c|c|c|c|}
\hline Corchorus olitorius L. & Tiliaceae & - & + & - \\
\hline Corchorus tridens L. & Tiliaceae & - & + & + \\
\hline Crotalaria goreensis G. et Perr. & Fabaceae & - & + & + \\
\hline Crotalaria mисronata Desv. & Fabaceae & - & + & + \\
\hline Ctenium newtonii Hack. & Poaceae & - & - & + \\
\hline Cymbopogon giganteus Chiov. & Poaceae & - & + & - \\
\hline Cymbopogon schoenanthus Spreng. & Poaceae & + & + & + \\
\hline Cyperus amabilis Vahhl. & Cyperaceae & - & - & + \\
\hline Cyperus esculentus L. & Cyperaceae & - & + & - \\
\hline Dactyloctenium aegyptium (L.) P. Beauv. & Poaceae & - & + & + \\
\hline Datura innoxia Mill. & Solanacea & - & - & + \\
\hline Digitaria horizontalis Willd. & Poaceae & - & + & + \\
\hline Diheteropogon hagerupii Hitchc. & Poaceae & - & - & + \\
\hline Elionorus elegans Kunth & Poaceae & + & + & + \\
\hline Eragrostis ciliaris (L.) R. Br. & Poaceae & - & - & + \\
\hline Eragrostis tenella (L.) P. Beauv. ex Roem et Schutt. & Poaceae & - & + & + \\
\hline Eragrostis tremula Hochst. ex Steud. & Poaceae & - & + & + \\
\hline Euclasta condylotricha (Hochst. ex Steud.) Stapf. & Poaceae & - & + & + \\
\hline Euphorbia convolvuloides Hochst. ex Benth. & Euphorbiaceae & - & + & + \\
\hline Euphorbia polycnemoides Hochst. ex Boiss. & Euphorbiaceae & - & + & + \\
\hline Evolvulus alsinoides $\mathrm{L}$. & Convolvulaceae & - & + & + \\
\hline Fimbrystilis hispidula (Vahl.) Kunth & Cyperaceae & + & + & + \\
\hline Gladiolus klathianus Hutch. & Iridaceae & - & - & + \\
\hline Grewia cissoides Hutch. et Dalz. & Tiliaceae & + & + & - \\
\hline Hackelochloa granularis (L.) O. Ktze. & Poaceae & - & + & + \\
\hline Hibiscus asper Hook. F. & Malvaceae & - & + & + \\
\hline Hyptis spicigera Lam. & Lamiaceae & - & + & + \\
\hline Indigofera colutea (Burm. f.) Merrill. & Fabaceae & + & + & + \\
\hline
\end{tabular}

2084 
G. YAMÉOGO et al. / Int. J. Biol. Chem. Sci. 7(5): 2078-2091, 2013

Indigofera dendroides Jacq.

Indigofera hirsuta $\mathrm{L}$.

Indigofera stenophylla Guill. et Perr.

Indigofera suffriticosa Mill.

Ipomoea eriocarpa $\mathrm{R}$. Br.

Ipomoea vagans Bak.

Kaempferia aethiopica (Schweinf.) Solms.Laub.

Kohautia senegalensis Cham. et Schlecht.

Lantana rhodesiensis Mold.

Lepidagathis anobrya Nees in DC.

Leptadenia hastata (Pers.) Decne

Leucas martinicensis (Jacq.) R. Br.

Lippia chevallieri Moldenke

Loudetia togoensis (Pilger) C.E. Hubb.

Microchloa indica (L.) P. Beauv.

Momordica charantia $\mathrm{L}$

Monechma ciliatum (Jacq.) Milne-Redh.

Paspalum orbiculare Forst.

Pennisetum pedicellatum Trin.

Peristrophe bicalyculata (Retz.) Nees

Polycarpaea corymbosa (L .) Lam.

Polygala arenaria Willd.

Polygala multiflora Poir.

Rottboellia exaltata L.

Sapium grahamii (Stapf.) Prain

Schizachyrium exile (Hochst.) Pilger

Schizachyrium sanguineum (Retz.) Alston

Schoenefeldia gracilis Kunth

\begin{tabular}{|c|c|c|c|}
\hline Fabaceae & - & + & + \\
\hline Fabaceae & - & + & - \\
\hline Fabaceae & - & + & - \\
\hline Fabaceae & + & + & + \\
\hline Convolvulaceae & - & + & - \\
\hline Convolvulaceae & + & + & + \\
\hline Zingiberaceae & - & - & + \\
\hline Rubiaceae & - & + & + \\
\hline Verbenaceae & - & + & + \\
\hline Acanthaceae & + & + & + \\
\hline Asclepiadaceae & - & + & + \\
\hline Lamiaceae & - & + & + \\
\hline Verbenaceae & - & + & + \\
\hline Poaceae & + & + & + \\
\hline Poaceae & + & + & + \\
\hline Cucurbitaceae & - & + & - \\
\hline Acanthaceae & + & + & - \\
\hline Poaceae & - & + & - \\
\hline Poaceae & + & + & + \\
\hline Acanthaceae & - & - & + \\
\hline Caryophyllaceae & - & + & + \\
\hline Polygalaceae & + & + & + \\
\hline Polygalaceae & - & + & - \\
\hline Poaceae & - & + & - \\
\hline Euphorbiaceae & - & - & + \\
\hline Poaceae & + & + & + \\
\hline Poaceae & + & + & - \\
\hline Poaceae & - & + & + \\
\hline
\end{tabular}

2085 
G. YAMÉOGO et al. / Int. J. Biol. Chem. Sci. 7(5): 2078-2091, 2013

Setaria pallide-fusca (Schum.) Stapf et Hubb.

Sida acuta Brum. F.

Sida alba L.

Sida rhombifolia L.

Sida urens L.

Sporobolus festivus Hochst. ex A. Rich.

Sporobolus pyramidalis P. Beauv.

Stachytarpheta angustilolia (Mill.) Vahl.

Striga hermontheca (Del.) Benth.

Striga macrantha Benth.

Stylochiton hypogaeus Lepr.

Stylochiton lancifolius Kotschy et Peyr

Stylosanthes erecta P. Beauv.

Tephrosia bracteolata Guill. Et Perr.

Tephrosia linearis (Willd.) Pers.

Tephrosia pedicellata Bak.

Tripogon minimus Hochst. ex Steud.

Vernonia ambigua Kotsc. et Peyr.

Vicoa leptoclada Dendy

Vigna ambacensis Welw. ex Bak.

Waltheria indica $\mathrm{L}$.

Wissadula amplissima (L.) R. E. Fries

Zornia glochidiata Reichb. ex DC.

Total

- espèces absentes

+ espèces présentes$$
\text { tespes présentes }
$$

Poaceae

Malvaceae

Malvaceae

Malvaceae

Malvaceae

Poaceae

Poaceae

Verbenaceae

Scrophulariaceae

Scrophulariaceae

Araceae

Araceae

Fabaceae

Fabaceae

Fabaceae

Fabaceae

Poaceae

Asteraceae

Asteraceae

Fabaceae

Sterculiaceae

Malvacea

Fabaceae

\begin{tabular}{lll}
- & + & + \\
- & + & + \\
+ & + & - \\
- & - & + \\
+ & + & - \\
+ & + & + \\
- & - & + \\
- & + & + \\
+ & + & + \\
- & - & + \\
- & + & - \\
- & - & + \\
- & - & + \\
- & + & + \\
- & + & - \\
+ & + & + \\
- & + & - \\
- & + & - \\
- & + & + \\
- & - & + \\
- & + & + \\
- & + & - \\
- & - & + \\
36 & 78 & 80 \\
\hline & &
\end{tabular}

eae


Phytomasse aérienne de la végétation herbacée

La phytomasse épigée des herbacées évaluée en fin de saison pluvieuse (octobre) dans les trois types de parcours étudiés était la suivante : $2205 \mathrm{~kg}$ de M.S /ha pour les buttes cuirassées, $2221,7 \mathrm{~kg}$ de M.S pour les glacis versant et $3334,7 \mathrm{~kg}$ de M.S pour les bas glacis. Il a été observé une augmentation de la phytomasse suivant la toposéquence.

Estimation de la capacité de charge et du bilan fourrager

Le Tableau 5 indique les valeurs de la capacité de charge et la capacité d'accueil pour chaque unité de parcours. Les capacités de charges théoriques des unités sont les plus importantes dans les bas glacis (0,73UBT/ha) par rapport aux deux autres unités où elles sont équivalentes. D'une façon générale la capacité d'accueil du terroir est importante et totalise 1286 UBT par an.

Par ailleurs il ressort que l'inventaire a permis de dénombrer 407 bovins, 624 ovins et 1529 caprins. Cela correspond à une biomasse animale de 604,32 UBT. Le bilan fourrager révèle donc un excédent de capacité d'accueil de près de 682 UBT.

Tableau 2: Nombre d'espèces par catégories fourragères et par type de parcours.

\begin{tabular}{lccc}
\hline Catégories fourragères & Buttes cuirassées & Glacis versants & Bas-glacis \\
\hline Poaceae & 15 & 25 & 26 \\
Légumineuses & 07 & 14 & 14 \\
Cyperaceae & 01 & 02 & 04 \\
Autres herbacées & 13 & 37 & 36 \\
Total & $\mathbf{3 6}$ & $\mathbf{7 8}$ & $\mathbf{8 0}$ \\
\hline
\end{tabular}

Tableau 3 : Contribution spécifique (en \%.) des espèces par catégorie fourragère et par type de parcours.

\begin{tabular}{lccc}
\hline Catégories fourragères & Buttes cuirassées & Glacis versants & Bas glacis \\
\hline Poaceae & 59,6 & 61,3 & 46,7 \\
Légumineuses & 16,8 & 9,7 & 9,8 \\
Cyperaceae & 4,4 & 2,4 & 2,7 \\
Autres herbacées & 19,2 & 26,7 & 40,8 \\
Total & $\mathbf{1 0 0}$ & $\mathbf{1 0 0}$ & $\mathbf{1 0 0}$ \\
\hline
\end{tabular}

Tableau 4: Valeur pastorale brute ( $\mathrm{VPb}$ en \%.) des espèces par catégories fourragères et par type de pâturage.

\begin{tabular}{lccc}
\hline Catégories fourragères & Buttes cuirassées & Glacis versants & Bas glacis \\
\hline Poaceae & 23,8 & 23,2 & 20,5 \\
Légumineuses & 5,7 & 3,2 & 3,7 \\
Cyperaceae & 0,0 & 0,0 & 0,1 \\
Autres herbacées & 3,0 & 4,1 & 4,8 \\
Total & $\mathbf{3 2 , 4}$ & $\mathbf{3 0 , 4}$ & $\mathbf{2 9 , 1}$ \\
\hline
\end{tabular}


Tableau 5: Capacité de charge et capacité d'accueil des différents types de parcours.

\begin{tabular}{|c|c|c|c|c|c|}
\hline \multirow[t]{2}{*}{ Types de pâturages } & \multirow{2}{*}{$\begin{array}{c}\text { Superficie } \\
\text { (ha) }\end{array}$} & \multirow{2}{*}{$\begin{array}{c}\text { Production de } \\
\text { phytomasse en kg } \\
\text { de MS/ha/an }\end{array}$} & \multicolumn{2}{|c|}{$\begin{array}{c}\text { Capacités de charge } \\
\text { (UBT/ha) }\end{array}$} & \multirow{2}{*}{$\begin{array}{c}\text { Capacité } \\
\text { d'accueil } \\
\text { (UBT) }\end{array}$} \\
\hline & & & $\begin{array}{c}\text { Saison } \\
\text { pluvieuse }\end{array}$ & $\begin{array}{c}\text { Toute } \\
\text { l'année }\end{array}$ & \\
\hline Buttes cuirassées & 173,7 & 2205 & 1,15 & 0,48 & 84 \\
\hline Glacis versants & 1237 & 2221,7 & 1,16 & 0,49 & 602 \\
\hline Bas glacis & 818,9 & 3334,7 & 1,74 & 0,73 & 600 \\
\hline
\end{tabular}

\section{DISCUSSION}

L'étude sur la végétation herbacée du terroir de Vipalogo montre que les pâturages naturels y sont constitués essentiellement de graminées annuelles dont les plus fréquentes sont Pennisetum pedicellatum Trin., Schoenefeldia gracilis Kunth, Setaria pallidefusca (Schum.) Stapf et Hubb. Andropogon pseudapricus Stapf., Loudetia togoensis (Pilger) C.E.Hubb., Elionurus elegans Kunth, Schizachyrium exile (Hochst.) Pilger, Brachiaria distichophylla (Trin.) Stapf et Microchlora indica (L.) P. Beauv.

Les légumineuses herbacées sont très peu représentées et seulement quelques unes d'entre elles sont appétées: Alysicarpus ovalifolius (Shum. et Thonn.) J. Leon., Zornia glochidiata Reichb. ex DC., Cassia mimosoïdes L., Cassia nigricans Vahl., Stylosanthes erecta P. Beauv., Vigna ambacensis Welw. Ex Bak. et Tephrosia pedicellata Bak.

Les principales graminées pérennes rencontrées sont Andropogon gayanus Kunth. , Andropogon ascinodis C.B.Cl., Cymbopogon giganteus Chiov. et Cymbopogon schoenanthus Spreng. De ces graminées pérennes, Andropogon gayanus, très appréciée par le bétail, se trouve être rare dans les parcours naturels du terroir. Cette rareté est essentiellement liée à la diminution ou à la disparition de la pratique de la jachère du fait de la forte anthropisation du milieu comme l'on fait remarquer de nombreux auteurs (César, 1992 ; Kaboré-Zoungrana, 1994; Yaméogo 1997), Cependant, de nombreuses études montrent bien l'importance de cette graminée pérenne dans l'alimentation du bétail (Haggar, 1970; Haggar et Ahmed,
1970 ; Sawadogo, 1990) et le rôle qu'elle joue comme indicateur de reprise de la fertilité des sols (Yaméogo, 1997).

Au regard de la diversité spécifique, on note une variation en fonction de la toposéquence. Il y a une diminution du nombre d'espèces quand on passe des basglacis aux buttes cuirassées. Cela obéit à une logique qui tient compte de plusieurs facteurs dont la qualité du sol, l'accessibilité par les animaux qui favorisent ou non la zoochorie, la dissémination des graines par l'écoulement des eaux de pluie, etc. L'ensemble de ces facteurs sont favorables aux glacis versants et aux bas-glacis, ce qui justifie le nombre élevé d'espèces en ces lieux.

Les espèces classées dans la catégorie «Autres herbacées » appartiennent à plusieurs familles (24 au total) et sont pour la plupart sans intérêt pastoral. Leur indice spécifique de qualité est pratiquement nul.

Ces résultats de la composition floristique des pâturages naturels de Vipalogo se rapprochent de ceux obtenus par Grouzis (1988) et Passini et al. (1989).

Au niveau des contributions spécifiques qui, en rappel, sont des expressions relatives de la biomasse des espèces, douze (12) espèces au sein des graminées marquent d'une façon significative le paysage floristique et de façon variable suivant les types de parcours. $\mathrm{Ce}$ sont Andropogon gayanus, Andropogon schirensis Hochst. Ex A.Rich., Andropogon fastigiatus Sw. Prod., Andropogon pseudapricus, Andropogon ascinodis, Aristida kerstingii Pilger, Schizachyrium exile, Diheteropogon hagerupii Hitchc., Sporobolus festivus Hochst. ex A Rich.., Pennisetum 
pedicellatum, Loudetia togoensis et Elionurus elegans Kunth.

En prenant en considération la valeur pastorale, les buttes cuirassées donnent les meilleurs résultats avec $32,40 \%$ suivies des glacis versants $30,41 \%$ et des bas-glacis $29,07 \%$. Cette diminution suivant la toposéquence est compréhensible si on tient compte du fait que suivant le même gradient les Poaceae et les légumineuses qui sont à forte valeur pastorale voient leur contribution spécifique diminuer au profit des Cyperaceae qui sont moins recherchées (Sawadogo et al, 2012). Mais d'une façon générale les valeurs pastorales globales des unités sont faibles par rapport aux valeurs indiquées pour les pâturages des zones nord soudaniennes. On suppose généralement pour cette région que les pâturages sont d'excellentes qualités si leur valeur pastorale atteint et dépasse les $65 \%$ (César, 2005). En plus de cette faiblesse des valeurs pastorales, les pâturages sont démunis en espèces pérennes qui indiquent qu'ils sont dégradés.

De nombreuses études effectuées sur la phytomasse aérienne des herbacées en zone soudano-sahélienne sur plusieurs types de pâturages traduisent bien la variabilité de la production suivant le type de sol et l'année (Zoungrana, 1991; Devineau et al., 1997 ; Devineau et al., 2010). Au regard des résultats obtenus, le terroir de Vipalogo connaît une exploitation très intensive des ressources végétales naturelles.

Le recensement du cheptel effectué dans les exploitations lors de l'étude a permis d'estimer les effectifs du cheptel ruminant du terroir à 604,31 UBT soit un besoin fourrager annuel d'environ 1379 tonnes de matière sèche. Par ailleurs, les productions de phytomasses des unités de végétation totalisent une disponibilité réelle en fourrage pour 1286 UBT. Cela équivaut à un surplus en disponibilité de fourrage par rapport au cheptel du terroir. En plus cette estimation ne tient pas compte de la fraction des ressources alimentaires composées des résidus de récoltes et des ligneux fourragers. Kiema et al. (2013) et Zampaligré et al. (2013) ont estimé que la contribution de cette fraction était très importante et pouvait varier de 20 à $70 \%$ dans l'alimentation des animaux selon les saisons. Cependant, il a également montré que les pâturages naturels sont communs et le cheptel des terroirs voisins et des transhumants y ont couramment accès; leurs effectifs peuvent atteindre près de $70 \%$ du cheptel au pâturage (Kiema et al., 2008). A cela s'ajoute les feux de brousse qui détruisent bien souvent une fraction importante durant la saison sèche. Le bilan fourrager réel est donc largement en deçà des estimations obtenues. Dans le contexte actuelle de nos systèmes d'élevage qui sont essentiellement traditionnelle il est difficile de cerner avec précision la réelle sur les terroirs. L'exploitation des pâturages prend en compte tous ces paramètres que les producteurs doivent maitriser, malheureusement ils ne sont pas suffisamment outillés pour cela.

\section{Conclusion}

Les résultats de cette étude montrent une disponibilité suffisante de la phytomasse herbacée par rapport au cheptel du terroir de Vipalogo. L'équilibre constaté entre le cheptel résident et la production de phytomasse devrait cependant prendre en compte la part du cheptel étranger transhumant et des terroirs voisins ainsi que la contribution des résidus de récolte et des ligneux fourrager. $\mathrm{La}$ disponibilité de chacune des ressources est saisonnière et cela dicte l'afflux des animaux en fonction de ces saisons. Il est donc opportun d'alléger la charge réelle du terroir par l'élaboration des règles locales de gestion des pâturages qui permettrait une répartition adéquate des troupeaux au niveau des parcours en tenant compte de leur potentiel de production et de leur capacité d'accueil.

Les glacis sont très sensibles et le surpâturage pourrait à terme contribuer à une dégradation irréversible.

En perspective, il serait souhaitable que cette étude puisse se poursuivre avec l'évaluation de la végétation ligneuse et la contribution des résidus de culture afin de pouvoir à terme proposer des actions 
concrètes dans le sens de l'amélioration de la gestion des ressources pastorales.

Les pâturages doivent être améliorés pour relever le niveau de valeur pastorale. $\mathrm{La}$ capacité technique et l'organisation des producteurs doivent être associées à ces améliorations pour une utilisation optimales et durable des pâturages. Le renforcement des capacités par la formation, l'organisation des structures locales de gestion sont autant d'outils à développer que l'amélioration des pâturages par des diverses techniques (pour améliorer les valeurs pastorales).

\section{REFERENCES}

Boudet G. 1984. Manuel sur les Pâturages Tropicaux et les Cultures Fourragères ( $4^{\mathrm{e}}$ édition révisée). MRECD/IEMVT : Paris ; 245 p.

Botoni-Liehoun E, Daget P, César J. 2006. Activités de pâturage, biodiversité et végétation pastorale dans la zone Ouest du Burkina Faso, Ressources alimentaires. Rév. Elev. Méd. Vét. Pays Trop., 59: 31-38.

César J. 1992. Etude de la production biologique des savanes de Côte-d'Ivoire et son utilisation par l'homme. Biomasse, valeur pastorale et production fourragère. Thèse de Doctorat de l'Université de Paris VI, Sciences naturelles, $642 \mathrm{p}$.

César J. 2005. L'Evaluation des Ressources Fourragères Naturelles. Productions Fourragères en Zone Tropicale. Production Animale en Afrique de l'Ouest; Synthèse. CIRDES/CIRAD: Bobo Dioulasso, Burkina Faso ; 12 p.

Daget $\mathrm{Ph}$, Poissonet J. 1971. Une méthode d'analyse phytologique des prairies. Critères d'application. Ann. Agron., 1: 41.

Devineau JL, Fournier A, Kaloga A. 1997. Les Sols et la Végétation de la Région de Bondoukuy, Sud-Ouest Burkinabè : Présentation Générale et Cartographie Préliminaire par Télédetection Satellitaire (SPOT). Editions de l'ORSTOM : Paris.
Devineau JL, Fournier A, Nignan S. 2010. Savanna fire regimes assessment with MODIS fire data: their relationship to land cover and plant species distribution in western Burkina Faso (West Africa). $J$. Arid Envi., 74: 1092-1101.

Djiteye M. 1988. Composition, structure et production des communautés végétales sahéliennes: application à la zone de Niono (Mali). Thèse Doct. Sc. de la vie, Université Paris-Sud, ORSAY, 150 p.

Floret C, LE Floc'h E, Pontanier R. 1983. Phytomasse et production végétale en Tunisie présaharienne. Acta Oecologica, Oecol. Plant., 4(18): 133-152.

Fontes J, Guinko S. 1995. Carte de la végétation et de l'occupation du sol au Burkina Faso (Notice explicative). Projet Campus. IDR, Université de Ouagadougou, $67 \mathrm{p}$.

Fournet J. 1983. Approche phytoécologique de l'amélioration fourragère des jachères dans un transect de la Péninsule Sud d'Haïti. Acta Oecologica, 4(3): 273-296.

Fournier A. 1982. Cycle saisonnier de la phyomasse herbacée dans les savanes de Ouango-Fitini. Ann. Univ. d'Abidjan, Série E. (Ecologie), XV: 63-94.

Godron M, Poissonet J, Poissonet P. 1967. Méthode d'étude des formations herbacées denses. Essai d'application à l'étude du dynamisme de la végétation. C.E.P.E. Montpellier, doc. $\mathrm{N}^{\circ} 35,28$ p.

Grouzis M. 1979. Structure, composition floristique et dynamique de la production de matière sèche des formations végétales sahéliennes (Mare d'Oursi ; HauteVolta). DGRST-ORSTOM. ACC lutte contre l'aridité dans l'Oudalan, p 59.

Grouzis M. 1988. Structure, Productivité et Dynamique des Systèmes Ecologiques Sahéliens (Mare d'Oursi, Burkina Faso). ORSTOM: Paris; 336 p.

Haggar JR. 1970. Seasonal production of Andropogon gayanus. I. Seasonal changes in yield compnents and chemical composition. J. Agric. Sci. Camb., 74: 487-494. 
Haggar JR, Ahmed MB. 1970. Seasonal production of Andropogon gayanus. II. Seasonal changes in digestibility and feed intake.J. Agric. Sci., Camb., 75: 369-373.

INSD. 2009. Recensement général de la population et de l'habitat 2006, Analyse des résultats définitifs, Thème II, Etat et structure de la population, $181 \mathrm{p}$.

Kaboré Zoungrana C, Zoungrana I, Sawadogo E. 1994. Variations saisonnières de la production de matière sèche et de la composition chimique d'Andropogon gayanus au Burkina Faso. Fourrages, 137: 61-74.

Kiema A, Nacro HB, Séogo SP. 2013. The use of the pasture areas by the cattle and goats in the Sahel region of Burkina Faso. Herald Journal of Agriculture and Food Science Research, 2(1): 52 - 62.

Kiema A, Nianogo AJ, Sanou OA, Sanou S. 2008. Caractéristiques des ressources fourragères dans les terroirs de Lelly (Zone Agro - pastorale) et N'Diahoye (Zone pastorale) au Nord du Burkina Faso. Revue Science et Technique, Série Science Naturelle et Agronomie, 29(1): 79-93.

Levang P, Grouzis M, 1980. Méthode d'étude de la biomasse herbacée de formations sahéliennes: application à la mare d'Oursi, Haute-Volta. Acta Oecologica, Oecol. Plant., 1(3): 231-244.

Millogo/Rasolodimby J. 2001. L'homme, le climat et les ressources alimentaires en période de crise de subsistance au cours du 20 ème siècle au Burkina Faso. Université de Ouagadougou, Thèse de Doctorat d'Etat, 248 p.

Passini MF, Delgadillo J, Salaza M. 1989. L'écosystème forestier de Barre-
Californie : composition floristique, variables écologiques principales, dynamique. Acta Oecologica, 10(3): 275293.

Poissonet P, César J. 1972. Structure spécifique de la strate herbacée dans la savane à Palmier Rônier de Lamto (Côte d'Ivoire). Ann. Univ. d'Abidjan, 5(1): 577-601.

Sawadogo E. 1990. Stade de développement, biomasse et valeur nutritive de quatre graminées fourragères: Brachiaria lata schumac (C.E. Hubbart), Pennisetum pedicellatum. Trin, Panicum anabaptistum stend. et Andropogon gayanus Kunth. Mémoire de fin d'étude IDR, Université de Ouagadougou.

Yameogo G. 1997. Etude diagnostique de la flore, de la végétation et du sol de jachères d'âges différents, dans le terroir de Thiougou (Burkina Faso). DEA, Université de Cocody, Côte d'Ivoire, 84p.

Yélémou B, Bationo B, Yaméogo G, Millogo-Rasolodimby J. 2007 .Gestion traditionnelle et usage de Piliostigma reticulatum (D.C.) Hochst., dans le Plateau central du Burkina Faso. Bois et Forêt des Tropiques, 291(1): 55-65.

Zoungrana I. 1991. Recherches sur les aires pâturées du Burkina Faso. Thèse d'Etat, Université de Bordeaux III, 290 p.

Zampaligré N. 2012. The role of ligneous vegetation for livestock nutrition in the sub Sahelian and Sudanian zones of West Africa: Potential effects of climate change. PhD thesis, University of Kassel, Germany, 100 p. 